\title{
REVISÃO DA QUALIDADE DO ESTUDO DE IMPACTO AMBIENTAL CENTRO LOGÍSTICO CAMPO GRANDE: UM ESTUDO DE CASO
}

\author{
Giovano Candiani \\ Universidade Federal de São Paulo, Campus Diadema \\ Departamento de Ciências Ambientais, Pós-Graduação em Análise Ambiental Integrada \\ Diadema, SP, Brasil \\ giovanocandiani@gmail.com \\ Andreza de Toledo Santana \\ Universidade Federal de São Paulo, Campus Diadema \\ Departamento de Ciências Ambientais, Graduação em Ciências Ambientais \\ Diadema, SP, Brasil \\ andrezasantanah@hotmail.com
}

\begin{abstract}
RESUMO
A revisão da qualidade dos Estudos de Impacto Ambiental é de fundamental importância para a eficiência do processo de Avaliação de Impacto Ambiental. Existem várias ferramentas para se fazer a revisão da qualidade dos estudos ambientais, entretanto, existe a necessidade de se comparar resultados obtidos com a aplicação de diferentes metodologias. Assim, o presente trabalho objetiva discutir a aplicabilidade de quatro ferramentas distintas para fins da revisão da qualidade em um EIA selecionado, o qual permita verificar a existência de similaridades e diferenças entre os resultados obtidos a partir da aplicação de tais ferramentas, vindo a contribuir tanto para o aperfeiçoamento, como para a padronização das práticas de avaliação da qualidade dos estudos de impacto ambiental. Os resultados encontrados indicam a existência de várias deficiências, destacando as relacionadas ao critério referente à análise dos impactos, mostrando a baixa qualidade do estudo ambiental avaliado. As distintas ferramentas permitiram conclusões semelhantes a respeito da qualidade do estudo. Conclui-se que estas ferramentas podem ser incorporadas pelos órgãos ambientais licenciadores, auxiliando os responsáveis na elaboração de pareceres técnicos para a avaliação e aprovação dos estudos ambientais.
\end{abstract}

Palavras-chave: Avaliação ambiental. Lista de verificação. Licenciamento ambiental.

\section{QUALITY REVIEW OF THE ENVIRONMENTAL IMPACT STUDY CAMPO GRANDE LOGISTICS CENTER: A CASE STUDY}

\begin{abstract}
The quality of Environmental Impact Studies is fundamentally important for the efficiency of the Environmental Impact Assessment process. There are several tools for reviewing the quality of environmental studies, however, there is a need to comparing results obtained by the usage of the different tools. The present work aims to discuss the applicability of four different tools for the purpose of quality review in a selected EIA, which allows to verify the existence of similarities and differences between the results obtained from the application of such tools, coming to contribute so much to the improvement, as well as for the standardization of quality assessment practices in environmental impact studies. Results pointed out several deficiencies, mainly related to the criteria matching the analysis of impacts which displayed low quality in the environmental study evaluated. Different tools brought similar conclusions regarding the quality of the following study. Finally, it concludes that tools under analysis could be incorporated by the environmental licensing agencies, as to assist technical opinions in evaluating and approving environmental studies.
\end{abstract}

Keywords: Environmental assessment. Verification list. Environmental licensing.

\section{INTRODUÇÃO}

A Avaliação de Impacto Ambiental (AIA) é um instrumento prévio de análise dos impactos ambientais de empreendimentos potencialmente causadores de significativos danos ao meio ambiente (SÁNCHEZ, 2013; AGRA FILHO, 2014; VERONEZ e MONTAÑO, 2017).

\begin{tabular}{llllll}
\hline Caminhos de Geografia & Uberlândia-MG & v. 21, n. 76 & Ago/2020 & p. 147-160 & Página 147
\end{tabular}


No contexto brasileiro, a AIA foi um instrumento instituído na Política Nacional de Meio Ambiente e regulamentado pela Resolução do Conselho Nacional de Meio Ambiente (CONAMA № 01 de 1986). A AIA é uma ferramenta de gestão ambiental importante para a conservação ambiental, porém existem muitas críticas quanto à sua eficiência no tocante aos seus procedimentos e práticas, principalmente em um Brasil repleto de carências estruturais e de recursos, como um todo (ALMEIDA et al. 2012; SÁNCHEZ, 2013; MONTAÑO et al., 2014; SCHOEN et al., 2016).

A AIA é constituída por duas vertentes: a político-jurídico-institucional (conjunto de normas ambientais) e a técnico-científica (estudos e avaliações de impactos ambientais). Além destas vertentes, é composta ainda pelo empreendedor, equipe técnica multidisciplinar e a sociedade - comunidade diretamente afetada - que se manifesta nas audiências públicas. Os componentes e atividades do processo de AIA apresentam a seguinte estrutura/fases: seleção e triagem (análise da proposta pelo órgão ambiental), determinação do escopo (termo de referência) e pós-aprovação (monitoramento) (PEREIRA et al., 2014).

Agra Filho (2014) salienta que, no processo de AIA, a etapa da definição do Termo de Referência (TR) é de fundamental importância, pois irá especificar os aspectos relevantes a serem enfatizados pelo Estudo de Impacto Ambiental (EIA).

A elaboração do EIA é a atividade cerne do processo de AIA, estabelecendo a base fundamental para análise da viabilidade ambiental do empreendimento. No EIA, os impactos ambientais são identificados e avaliados em termos de significância e magnitude, incluindo-se os meios físico, biológico e antrópico. São estabelecidas também as medidas mitigadoras e compensatórias, bem como o plano básico de gestão ambiental (SÁNCHEZ, 2013).

Veronez e Montaño (2017) apontam que a qualidade do EIA é fundamental para a efetividade do sistema de AIA e, portanto, essencial que sejam realizadas pesquisas para a análise da qualidade dos estudos de impactos ambientais, identificando-se pontos fracos e oportunidades de melhoria.

Lee e Colley (1992) reforçam que um bom ElA precisa ter forma e conteúdo adequados e que apresentem aos interessados, de maneira apropriada, constatações e conclusões que efetivamente cubram as principais tarefas da avaliação, emprego de métodos coerentes de diagnóstico, análise e comunicação.

$\mathrm{Na}$ literatura encontram-se relatadas várias deficiências, tais como: ausência ou fragilidade dos termos de referência, falta de alternativas locacionais e tecnológicas, medidas mitigadoras muito genéricas, planos de monitoramento superficiais, carência de procedimentos técnicos adequados para identificar e prever impactos, omissões e previsões subestimadas destes impactos, imprecisões de critérios para definir áreas afetadas, áreas de influências e populações atingidas, EIAs tendenciosos, diagnóstico ambiental deficitário e Rima incompleto e/ou com linguagem inadequada à compreensão do público (ALMEIDA et al., 2012; SÁNCHEZ, 2013; MONTAÑO et al., 2014; ALMEIDA e MONTAÑO, 2017; VERONEZ e MONTAÑO, 2017; MAZZEI; MARANGONI; OLIVEIRA, 2018; SILVA JUNIOR; ALVARENGA; GARCIA, 2018; ALMEIDA et al., 2019).

A análise da qualidade dos EIAs depende também de ferramentas adequadas, sendo comum a aplicação de listas de verificação durante esta tarefa (HICKIE e WADE, 1998; BONDE e CHERP, 2000; PÕDER e LUKKI, 2011; SÁNCHEZ, 2013; MONTAÑO et al., 2014; MOUNIR, 2015; VERONEZ e MONTAÑO, 2017; BERTUOLA e CANDIANI, 2018).

Lee e Colley (1992) desenvolveram um procedimento de análise baseado na avaliação de conteúdo de cada um dos principais componentes normalmente encontrados em um EIA. Esta técnica consiste em dividir a análise do EIA em quatro seções: (i) descrição do projeto e do ambiente afetado, (ii) identificação e avaliação de impactos chave, (iii) consideração de alternativas e medidas mitigadoras e (iv) comunicação dos resultados. Cada seção é subdividida em categorias que, por sua vez, são subdivididas em subcategorias - estas com maior grau de detalhamento. Este método também emprega critérios para atribuição de conceitos à cada subcategoria e área, bem como de uma nota geral ao EIA. Os critérios de notas variam de $\mathrm{A}$ a $\mathrm{F}$, em que $\mathrm{A}$ corresponderá à melhor nota e $\mathrm{F}$, à pior.

Glasson, Therivel e Chadwick, (2005) criaram uma lista de verificação organizada em oito seções, cada uma delas contendo itens, ou perguntas, a serem avaliados segundo uma notação de $A$ à $F$, em molde

$\begin{array}{llllll}\text { Caminhos de Geografia } & \text { Uberlândia-MG } & \text { v. 21, n. 76 } & \text { Ago/2020 } & \text { p. 147-160 Página } 148\end{array}$


similar ao de Lee e Colley (1992): (i) descrição do projeto, (ii) descrição do ambiente, (iii) escopo, consulta e identificação dos impactos, (iv) previsão e avaliação de impactos, (v) alternativas, (vi) mitigação e monitoramento, (vii) resumo não técnico e (viii) organização e apresentação da informação.

Pinho, Maia e Monterroso (2007) apresentaram ferramenta de avaliação composta por 12 seções, contemplando os seguintes critérios: (i) escopo, (ii) caracterização do projeto, (iii) inclusão de alternativas, (iv) diagnóstico ambiental, (v) identificação de impactos, (vi) descrição dos impactos previstos, (vii) medidas mitigadoras, (viii) monitoramento, (ix) dificuldades técnicas e ausência de informações, (x) participação social, (xi) apresentação dos resultados e (xii) Rima. Para cada um destes critérios existe composição de subcritérios, em que são atribuídas três faixas de escala: baixo (quando o critério for considerado incompleto e tecnicamente insatisfatório), médio (quando o critério for considerado razoável ou apenas satisfatório) e alto (quando o critério for considerado completo e muito satisfatório). Atribui-se peso variável entre 0,0 a 3,0 na ponderação dos critérios, ora categorizados em seis agrupamentos distintos, indo de I a VI ("muito fraco" a "muito bom").

É possível identificar muitos estudos aplicando diferentes ferramentas e critérios de análise da qualidade dos estudos de impacto ambiental, entretanto, nem todos fazem comparações dos resultados obtidos com a aplicação destas diferentes ferramentas e critérios.

Neste contexto, o presente trabalho objetiva discutir a aplicabilidade de quatro ferramentas distintas para fins da revisão da qualidade em um EIA selecionado, o qual permita verificar a existência de similaridades e diferenças entre os resultados obtidos a partir da aplicação de tais ferramentas, vindo a contribuir tanto para o aperfeiçoamento, como para a padronização das práticas de avaliação da qualidade dos estudos de impacto ambiental.

\section{METODOLOGIA}

Para a análise da qualidade, o EIA selecionado neste estudo foi o Centro Logístico Campo Grande, um empreendimento a ser implantado na Região da Vila de Paranapiacaba, distrito do município de Santo André-SP.

A seleção deste EIA-Rima deu-se pelo aspecto polêmico da possível implantação de um complexo logístico no estado de São Paulo, na Serra do Mar, ao lado da vila histórica de Paranapiacaba (tombada como patrimônio cultural), envolvendo impactos ambientais negativos, uma vez que se trata de área de remanescentes de Mata Atlântica com presença de Unidades de Conservação e conflitos com a população local (TORRES; RAMOS; GONÇALVES, 2019).

O processo de solicitação da licença ambiental para implantação deste empreendimento na Região encontra-se em curso desde 2017. Apesar do objeto de licenciamento não estar propriamente localizado na Vila, encontra-se muito próximo a ela, a $5 \mathrm{~km}$ de distância, apresentando, portanto, elevado potencial para causar significativos impactos socioambientais negativos em toda a área.

Além de patrimônio cultural, a Vila possui um patrimônio histórico singular, reconhecido através dos tombamentos federal, estadual e municipal, e se encontra em constante busca pelo desenvolvimento sustentável. O empreendimento não se adequa ao caráter de desenvolvimento local, ora relacionado às atividades de ecoturismo, economia solidária e conservação ambiental, perfazendo óbvio contexto de elevada fragilidade ambiental.

\section{ÁREA DE ESTUDO}

A Vila de Paranapiacaba (Figura 1) é um distrito do município de Santo André-SP, representando $48 \%$ de sua área total. Encontra-se na Serra do Mar, em uma área de mananciais e Mata Atlântica conservada, e seu nome significa, em tupi-guarani, "local de onde se vê o mar". Paranapiacaba é atualmente região turística de grande importância ambiental, possuindo três Unidades de Conservação na Região: o Parque Natural Municipal Nascentes de Paranapiacaba, a Reserva Biológica do Alto da Serra de Paranapiacaba e o Parque Estadual da Serra do Mar (RICCOMINI, 2018).

\begin{tabular}{llllll}
\hline Caminhos de Geografia & Uberlândia-MG & v. 21, n. 76 & Ago/2020 & p. 147-160 & Página 149
\end{tabular}


Figura1 - Vila de Paranapiacaba, Santo André (SP): Macrozoneamento ambiental.

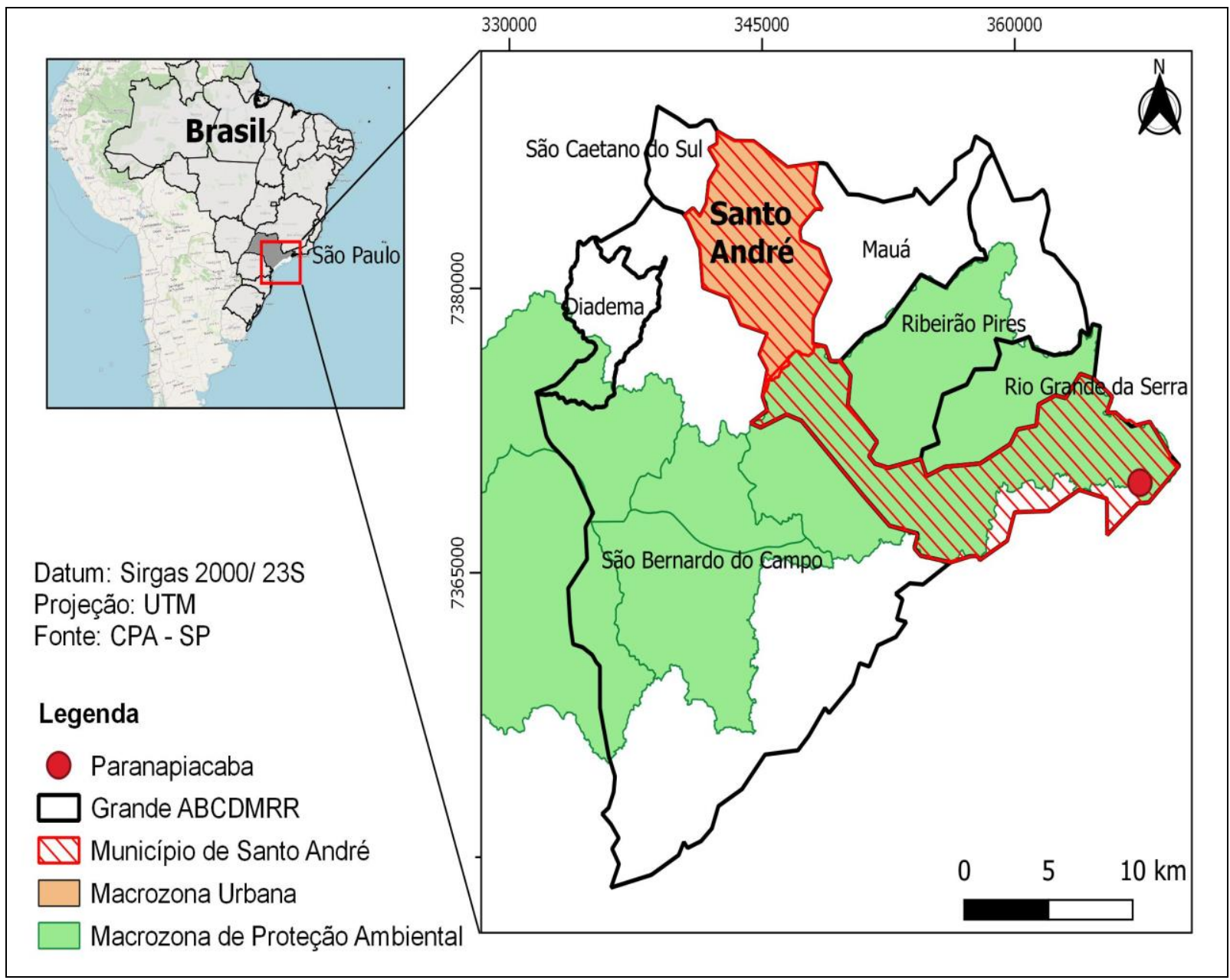

Fonte - Elaborado pelos autores (2020).

A Vila tem sua origem estritamente relacionada à Estrada de Ferro Santos-Jundiaí, inaugurada em 1867 pela São Paulo Railway Company. A companhia construiu a Estação Alto da Serra no ano seguinte com o intuito de manter funcionários no local para a complementação das obras, quando então passou a chamar-se Paranapiacaba, em 1945. A população da Vila é heterogênea e sua situação social é complexa, sendo formada por descendentes das famílias de ferroviários, que ocupam a Vila desde sua fundação e, mais recentemente, por famílias de situação social frágil que invadiram casas abandonadas. A Prefeitura de Santo André assumiu e fomentou o turismo local, melhorando muito a situação da Vila, porém ainda hoje a população possui baixa renda e enfrenta uma série de precariedades nas áreas da saúde, educação e infraestrutura (RICCOMINI, 2018).

\section{CARACTERIZAÇÃO DO EMPREENDIMENTO}

O Centro Logístico Campo Grande (CLCG) é um empreendimento que atualmente encontra-se em processo de licenciamento ambiental para obtenção, junto à CETESB (Companhia Ambiental do Estado de São Paulo), da licença prévia para a implantação de um condomínio destinado a abrigar atividades de logística de cargas, utilizando como meios de transporte a ferrovia e a rodovia adjacentes à propriedade, tendo por proponente a empresa Fazenda Campo Grande Empreendimentos e Participações Ltda. e por responsável pelo EIA/Rima a CPEA - Consultoria, Planejamento e Estudos Ambientais Ltda.

O empreendimento distribui-se em três glebas localizadas à margem da ferrovia Santos-Jundiaí, no bairro Campo Grande, distrito de Paranapiacaba, no município de Santo André (SP). A área total das glebas soma 468 ha, sendo que no projeto original, descrito no ElA/Rima, 91 hectares seriam efetivamente ocupados (CPEA, 2017).

$\begin{array}{llllll}\text { Caminhos de Geografia } & \text { Uberlândia-MG } & \text { v. 21, n. 76 } & \text { Ago/2020 } & \text { p. 147-160 } & \text { Página } 150\end{array}$




\section{PROCEDIMENTO METODOLÓGICO}

O procedimento metodológico para análise da qualidade do EIA foi baseado na aplicação de quatro ferramentas de revisão distintas: "Lee e Colley Review Package" (Lee e Colley, 1992), "Environmental Impact Statement Review Package" (Glasson; Therivel; Chadwick, 2005), "Guia para Análise Técnica de Estudos de Impacto Ambiental" (Sánchez, 2013) e o modelo proposto por Pinho, Maia e Monterroso (2007), contido na "Avaliação da Qualidade de Estudos de Impacto Ambiental de Pequenas Centrais Hidrelétricas".

A seleção destes métodos de análise deu-se pela ampla e consagrada aplicação dos mesmos na literatura internacional e brasileira.

O método "Lee e Colley Review Package" é amplamente aplicado e difundido na literatura internacional (Lee e Colley, 1992) e adota critérios baseados em boas práticas na análise de EIAs.

A metodologia baseia a análise de um EIA em quatro seções principais: (i) Descrição do projeto e da linha de base, (ii) Identificação e avaliação dos principais impactos, (iii) Alternativas e mitigação e (iv) Comunicação dos resultados.

Estas 4 seções dividem-se em 17 categorias e, a partir destas, em 52 subcategorias. Os critérios são dispostos em uma estrutura hierárquica da avaliação, a qual parte da base da pirâmide (nível mais simples) ao topo (nível mais complexo). A escala varia de $\mathrm{A}$ (bem realizado) a $\mathrm{F}$ (muito insatisfatório).

O método "Environmental Impact Statement Review Package" foi criado por Glasson, Therivel e Chadwick (2005). Esta ferramenta compreende 96 critérios organizados em 8 seções principais, sejam elas: (i) Descrição do empreendimento, (ii) Descrição do ambiente, (iii) Escopo, consulta pública e identificação de impactos, (iv) Previsão e avaliação dos impactos, (v) Alternativas, (vi) Mitigação e monitoramento, (vii) Resumo não técnico (Rima) e (viii) Organização e apresentação das informações.

Aqui, também cada critério é avaliado em termos de qualidade, sendo utilizada escala de notação que varia de $\mathrm{A}$ a $\mathrm{F}$, em que $\mathrm{A}$ corresponderá à melhor nota e $\mathrm{F}$, à pior.

O método "Guia para Análise Técnica de Estudos de Impacto Ambiental" proposto por Sánchez (2013) para revisão da qualidade do EIA, consiste em uma ferramenta brasileira formada por 13 seções principais, divididas em 30 subitens os quais, por sua vez, formam 112 critérios. As seções são: (i) Sumário, (ii) Introdução, (iii) Informações gerais, (iv) Caracterização do empreendimento, (v) Diagnóstico ambiental, (vi) Análise dos impactos, (vii) Programas de gestão ambiental, (viii) Síntese e conclusões, (ix) Referências bibliográficas, (x) Glossário de termos técnicos, (xi) Descrição da equipe técnica, (xii) Anexos e (xiii) Relatório de impacto ambiental.

A análise dos critérios inclui um checklist ("sim ou não") e a atribuição de conceitos a estes critérios, os quais variam entre "completo", "aceitável" ou "inadequado".

O método "Avaliação da Qualidade de Estudos de Impacto Ambiental de Pequenas Centrais Hidrelétricas" (PCHs) desenvolvido por Pinho, Maia e Monterroso (2007) é formado por 12 seções contemplando os seguintes critérios associados ao EIA: (i) Escopo, (ii) Caracterização do projeto, (iii) Inclusão de alternativas, (iv) Diagnóstico ambiental, (v) Identificação de impactos, (vi) Descrição dos impactos previstos, (vii) Medidas mitigadoras, (viii) Monitoramento, (ix) Dificuldades técnicas e ausência de informações, (x) Participação social, (xi) Apresentação dos resultados e (xii) Rima.

Estes critérios são subdivididos em 47 subcritérios à cada qual, consideradas três faixas de escalas, é atribuída uma classificação: baixo (quando o critério for considerado incompleto e tecnicamente insatisfatório), médio (quando o critério for considerado razoável ou apenas satisfatório) e alto (quando o critério for considerado completo e muito satisfatório).

Atribui-se peso na ponderação de cada subcritério individualmente, o qual varia de 0,0 a 3,0 e, após, categorizando-se em seis agrupamentos distintos, como na sequência: I. Muito fraco $(0-0,4)-$ quando a maioria dos subcritérios não foram contempladas ou foram deficientes, II. Fraco $(0,5-0,9)$ - quando uma quantidade grande de subcritérios não foi contemplada e alguns outros apresentam deficiências, III. Regular $(1,0-1,4)$ - quando parte dos subcritérios foi contemplada, mas outra parte permaneceu abaixo de padrões satisfatórios, IV. Satisfatório $(1,5-1,9)$ - quando apenas uma pequena parte dos subcritérios não foi contemplada, mas outra parte significativa atendeu níveis satisfatórios, V. Bom $(2,0-2,4)-$ quando todos os subcritérios foram contemplados e a maioria deles apresenta níveis satisfatórios e VI. Muito bom $(2,5-3,0)$ - quando todos os subcritérios foram contemplados em nível satisfatório. Após a atribuição das notas, calculam-se as médias das seções.

\begin{tabular}{llllll}
\hline Caminhos de Geografia & Uberlândia-MG & v. 21, n. 76 & Ago/2020 & p. 147-160 & Página 151
\end{tabular}


A atribuição dos conceitos aos itens (critérios) das quatro ferramentas avaliadas, foi estabelecido da seguinte maneira: avaliação separadamente por três avaliadores (equipe interdisciplinar), com posterior comparação e discussão, objetivando-se estabelecer ajuste e alinhamento consensual dos resultados obtidos (VERONEZ e MONTAÑO, 2017).

\section{RESULTADOS E DISCUSSÃO}

Os resultados obtidos com a aplicação das quatro ferramentas de análise da qualidade do EIA e avaliados no presente trabalho são sistematizados no Quadro 1.

Em termos gerais, nenhuma ferramenta utilizada considerou o EIA analisado adequado, sugerindo-se a sua baixa qualidade.

A tomada de decisão por parte de um órgão ambiental licenciador, baseado em informações de qualidade insatisfatória, prejudica em muito o processo de avaliação e licenciamento ambiental (VERONEZ e MONTAÑO, 2017).

Quadro 1 - Revisão da qualidade do EIA pelos métodos empregados no presente trabalho.

\begin{tabular}{|c|c|c|}
\hline Métodos & Critérios & Conceito Geral \\
\hline $\begin{array}{l}\text { Lee e Colley } \\
\text { (1992) }\end{array}$ & $\begin{array}{l}\text { 1. Descrição do empreendimento e da linha de base } \\
\text { 2. Identificação e avaliação dos principais impactos } \\
\text { 3. Alternativas e mitigação } \\
\text { 4. Comunicação dos resultados } \\
\text { Conceito final geral }\end{array}$ & $\begin{array}{c}\text { D } \\
\text { E } \\
\text { D } \\
\text { C } \\
\text { D (insatisfatório) }\end{array}$ \\
\hline $\begin{array}{c}\text { Glasson, } \\
\text { Therivel e } \\
\text { Chadwick } \\
(2005)\end{array}$ & $\begin{array}{l}\text { 1. Descrição do empreendimento } \\
\text { 2. Descrição do ambiente } \\
\text { 3. Escopo, consulta pública e identificação de impactos } \\
\text { 4. Previsão e avaliação dos impactos } \\
\text { 5. Alternativas } \\
\text { 6. Mitigação e monitoramento } \\
\text { 7. Resumo não técnico (Rima) } \\
\text { 8. Organização e apresentação das informações } \\
\text { Conceito final geral }\end{array}$ & $\begin{array}{l}\text { C } \\
\text { C } \\
\text { D } \\
\text { D } \\
\text { D } \\
\text { D } \\
\text { C } \\
\text { C } \\
\text { D (insatisfatório) }\end{array}$ \\
\hline Sánchez (2013) & $\begin{array}{l}\text { 1. Sumário } \\
\text { 2. Introdução } \\
\text { 3. Informações gerais } \\
\text { 4. Caracterização do empreendimento } \\
\text { 5. Diagnóstico ambiental } \\
\text { 6. Análise dos impactos } \\
\text { 7. Programas de gestão ambiental } \\
\text { 8. Síntese e conclusões } \\
\text { 9. Rima } \\
\text { Conceito final geral }\end{array}$ & $\begin{array}{l}\text { Completo } \\
\text { Completo } \\
\text { Inadequado } \\
\text { Inadequado } \\
\text { Inadequado } \\
\text { Inadequado } \\
\text { Inadequado } \\
\text { Inadequado } \\
\text { Aceitável } \\
\text { (Inaceitável) }\end{array}$ \\
\hline $\begin{array}{c}\text { Pinho, Maia e } \\
\text { Monterroso } \\
\text { (2007) }\end{array}$ & $\begin{array}{l}\text { 1. Sumário } \\
\text { 2. Caracterização do projeto } \\
\text { 3. Inclusão de alternativas } \\
\text { 4. Diagnóstico ambiental } \\
\text { 5. Identificação de impactos } \\
\text { 6. Descrição dos impactos previstos } \\
\text { 7. Medidas mitigadoras } \\
\text { 8. Monitoramento } \\
\text { 9. Dificuldades técnicas e ausência de informações } \\
\text { 10. Participação social } \\
\text { 11. Apresentação dos resultados } \\
\text { 12. Rima } \\
\text { Conceito final geral }\end{array}$ & $\begin{array}{c}\text { 1,5 (Satisfatório) } \\
\text { 1,2 (Regular) } \\
\text { 0,9 (Fraco) } \\
\text { 1,4 (Regular) } \\
\text { 1,1 (Regular) } \\
\text { 1,2 (Regular) } \\
\text { 0,6 (Fraco) } \\
\text { 1,3 (Regular) } \\
\text { 0,5 (Fraco) } \\
\text { 0,2 (Muito fraco) } \\
\text { 1,6 (Satisfatório) } \\
\text { 2,0 (Bom) } \\
\mathbf{1 , 1} \text { (Regular) }\end{array}$ \\
\hline
\end{tabular}

Fonte - Elaborado pelos autores (2020). 
A ferramenta de Lee e Colley (1992) resultou em um conceito final geral "insatisfatório" em termos de qualidade do EIA analisado (Quadro 1), apresentando omissões importantes e muitos critérios inadequados. O critério 2 (identificação e avaliação dos principais impactos) foi o que obteve a menor nota (E), com omissões e inadequações significativas, aspecto que prejudica em muito a qualidade do estudo, pois este critério é composto por categorias de fundamental importância, tais como: a definição e identificação dos impactos, e a previsão e análise da magnitude e significância dos impactos. A área 4 (comunicação dos resultados) apresentou o melhor desempenho dentre as quatro áreas analisadas, obtendo conceito $\mathrm{C}$ (satisfatório), apresentando omissões e pontos inadequados. O critério 4 refere-se a itens como a apresentação do empreendimento à comunidade e a elaboração do Rima.

O método proposto por Glasson, Therivel e Chadwick (2005) também apresentou conceito final geral insatisfatório (Quadro 1), com omissões e inadequações. Critérios fundamentais para a qualidade de um EIA foram considerados negativos, como por exemplo: consulta pública, identificação de impactos, previsão e avaliação dos impactos, alternativas locacionais e tecnológicas e mitigação e monitoramento.

A análise proposta por Sánchez (2013) resultou em um conceito final geral inaceitável (Quadro 1), com vários critérios apresentando omissões significativas, necessitando de informações complementares e adequações. Dos 9 critérios gerais da metodologia de análise, 6 foram conceituados como inadequados, incluindo o item referente à análise dos impactos ambientais (critério 6).

Já a ferramenta proposta por Pinho, Maia e Monterroso (2007) determinou um conceito final geral regular (Quadro 1), contemplando critérios abaixo dos padrões satisfatórios. Critérios importantes, como a caracterização do projeto, o diagnóstico ambiental, a identificação e a descrição dos impactos e monitoramento foram considerados regulares, ou seja, inadequados. Os critérios relacionados à inclusão de alternativas locacionais e tecnológicas e às medidas mitigadoras foram classificados como fracos. Quanto à participação social, item importante considerando-se que este critério, de certa maneira, representa a comunidade diretamente afetada pelo empreendimento, foi categorizado como muito fraco. Todos estes aspectos negativos reforçam a percepção da baixa qualidade do EIA analisado.

Buscando aprimoramento em relação à avaliação da qualidade do EIA estudado, o Quadro 2 mostra a análise por categoria individual em relação aos resultados, segundo a metodologia de Lee e Colley (1992), permitindo analisar os critérios satisfatórios (em verde) e os insatisfatórios (em vermelho). Nota-se que $35 \%$ dos critérios se caracterizam como satisfatórios e $65 \%$ são insatisfatórios. O destaque negativo apresenta-se em relação a Área 2 (identificação e avaliação de impactos-chave), em que nenhum critério foi considerado satisfatório.

Este foi um resultado importante, pois mostrou que os critérios relacionados à identificação e avaliação dos impactos ambientais precisam ser aperfeiçoados. Veronez e Montaño (2017) também encontraram resultados mais insatisfatórios relacionados aos critérios de identificação e avaliação dos impactos ao estudarem a qualidade de EIAs no estado do Espírito Santo no período de 2007 a 2013.

Muitos dos critérios elencados como fracos foram apontados anteriormente em distintos trabalhos na literatura, destacando-se os estudos realizados por ALMEIDA et al. (2012); MONTAÑO et al. (2014); VERONEZ e MONTAÑO (2017); BERTUOLA e CANDIANI (2018).

Em 2004, uma equipe de analistas do Ministério Público Federal (MPF) realizou um trabalho importantíssimo de análise relativo à qualidade dos EIAs no Brasil. Os resultados foram publicados em um compilado informativo com as insuficiências apontadas neste estudo (MPF, 2004).

Ainda em relação ao estudo do Ministério Público Federal, acima, foram relatadas muitas deficiências, destacando-se as seguintes: ausência de proposição de alternativas locacionais e tecnológicas, desconsideração da bacia hidrográfica na delimitação das áreas de influência do projeto, delimitação das áreas de influência sem alicerce nas características e vulnerabilidades dos ambientes naturais e nas realidades sociais regionais, prazos insuficientes para a realização de pesquisas de campo no diagnóstico ambiental, ausência ou insuficiência de informações sobre as metodologias utilizadas, ausência de mapas temáticos, ausência ou insuficiência de dados quantitativos sobre a vegetação e a fauna da área do projeto, conhecimento insatisfatório dos modos de vida de coletividades socioculturais singulares e de suas redes intercomunitárias das populações locais, ausência de estudos orientados pela ampla acepção do conceito de patrimônio cultural, não identificação de determinados impactos, omissão de dados e/ou justificativas quanto à metodologia utilizada para arrogar pesos aos atributos dos impactos, tendência à minimização ou subestimação dos impactos negativos e à supervalorização dos impactos positivos, proposição de medidas que não são a solução para a mitigação do impacto, ausência de avaliação da

\begin{tabular}{llllll}
\hline Caminhos de Geografia & Uberlândia-MG & v. 21, n. 76 & Ago/2020 & p. 147-160 & Página 153
\end{tabular}


eficiência das medidas mitigadoras propostas, ausência de proposição de programa de monitoramento de impactos específicos, RIMA incompleto e emprego de linguagem inadequada à compreensão do público.

Quadro 2 - Notas atribuídas ao estudo analisado com os critérios de Lee e Colley (1992).

\begin{tabular}{|c|c|c|}
\hline \multirow{5}{*}{$\begin{array}{l}\text { Área } 1 \\
\text { Descrição do projeto e do ambiente } \\
\text { afetado }\end{array}$} & 1.1. Descrição do projeto. & \\
\hline & 1.2. Descrição do local. & \\
\hline & 1.3. Resíduos. & \\
\hline & \multicolumn{2}{|l|}{ 1.4. Descrição do ambiente. } \\
\hline & \multicolumn{2}{|l|}{ 1.5. Condições da linha de base. } \\
\hline \multirow{5}{*}{$\begin{array}{l}\text { Área } 2 \\
\text { Identificação e avaliação de impactos- } \\
\text { chave }\end{array}$} & \multicolumn{2}{|l|}{ 2.1. Definição dos impactos. } \\
\hline & \multicolumn{2}{|l|}{ 2.2. Identificação dos impactos. } \\
\hline & \multicolumn{2}{|l|}{ 2.3. Escopo. } \\
\hline & \multicolumn{2}{|l|}{$\begin{array}{l}\text { 2.4. Previsão da magnitude do } \\
\text { impacto. }\end{array}$} \\
\hline & \multicolumn{2}{|l|}{$\begin{array}{l}\text { 2.5. Avaliação de significância dos } \\
\text { impactos. }\end{array}$} \\
\hline \multirow{3}{*}{$\begin{array}{l}\text { Área } 3 \\
\text { Consideração de alternativas e medidas } \\
\text { mitigadoras }\end{array}$} & \multicolumn{2}{|l|}{ 3.1. Alternativas. } \\
\hline & \multicolumn{2}{|l|}{$\begin{array}{l}\text { 3.2. Escopo e efetividade das } \\
\text { medidas mitigadoras. }\end{array}$} \\
\hline & \multicolumn{2}{|l|}{ 3.3. Compromisso com a mitigação. } \\
\hline \multirow{6}{*}{$\begin{array}{l}\text { Área } 4 \\
\text { Comunicação dos resultados }\end{array}$} & \multicolumn{2}{|l|}{ 4.1. Layout. } \\
\hline & \multicolumn{2}{|l|}{ 4.2. Apresentação. } \\
\hline & \multicolumn{2}{|l|}{ 4.3. Ênfase. } \\
\hline & \multicolumn{2}{|l|}{ 4.4. Resumo não técnico (RIMA). } \\
\hline & \multicolumn{2}{|l|}{ Escala das Notas } \\
\hline & 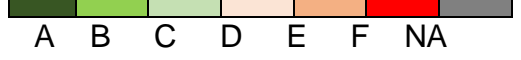 & \\
\hline
\end{tabular}

Fonte - Elaborado pelos autores (2020).

O Quadro 3 representa a análise em relação aos resultados por categoria individual segundo os critérios de Glasson, Therivel e Chadwick (2005). Quanto às notas dos critérios, as insatisfatórias (61\%) superaram as satisfatórias (39\%). A seção 2 (descrição do meio ambiente) foi a que apresentou melhor desempenho. Os piores resultados foram atribuídos às seções relacionadas à: identificação e avaliação dos impactos, alternativas (locacionais e tecnológicas) e mitigação e monitoramento dos impactos (seções 3, 4, 5 e 6). Almeida et al. (2012) aplicaram este mesmo método e obtiveram resultados similares, destacando-se notas insatisfatórias paras estas mesmas seções mencionadas.

$\begin{array}{llllll}\text { Caminhos de Geografia } & \text { Uberlândia-MG } & \text { v. 21, n. 76 } & \text { Ago/2020 } & \text { p. 147-160 } & \text { Página } 154\end{array}$


Quadro 3 - Notas atribuídas ao estudo analisado com os critérios de Glasson, Therivel e Chadwick (2005).

\begin{tabular}{|c|c|}
\hline \multirow[t]{4}{*}{$\begin{array}{l}\text { Seção } 1 \\
\text { Descrição do empreendimento }\end{array}$} & $\begin{array}{l}\text { Principais características do projeto (objetivos, } \\
\text { descrição etc.). }\end{array}$ \\
\hline & $\begin{array}{l}\text { Requisitos espaciais (área ocupada, localização } \\
\text { etc.). }\end{array}$ \\
\hline & $\begin{array}{l}\text { Entradas do projeto (natureza, quantidades de } \\
\text { materiais, funcionários, meios de transporte etc.). }\end{array}$ \\
\hline & Resíduos e emissões. \\
\hline \multirow[t]{2}{*}{$\begin{array}{l}\text { Seção } 2 \\
\text { Descrição do meio ambiente }\end{array}$} & $\begin{array}{l}\text { Descrição da área ocupada e circundante ao projeto } \\
\text { (ambiente afetado, uso da terra etc.). }\end{array}$ \\
\hline & $\begin{array}{l}\text { Condições de linha de base (componentes do } \\
\text { ambiente afetado, condições ambientais futuras, } \\
\text { planos e políticas etc.). }\end{array}$ \\
\hline \multirow{2}{*}{$\begin{array}{l}\text { Seção } 3 \\
\text { Escopo, consulta e identificação } \\
\text { de impacto }\end{array}$} & Escopo e consulta pública. \\
\hline & Identificação do impacto. \\
\hline \multirow{3}{*}{$\begin{array}{l}\text { Seção } 4 \\
\text { Predição e avaliação de impactos }\end{array}$} & Previsão da magnitude dos impactos. \\
\hline & Métodos e dados. \\
\hline & Avaliação da significância do impacto. \\
\hline $\begin{array}{l}\text { Seção } 5 \\
\text { Alternativas }\end{array}$ & $\begin{array}{l}\text { Alternativas locacionais e tecnológicas do projeto } \\
\text { (alternativas realistas, genuínas, justificativas das } \\
\text { escolhas etc.). }\end{array}$ \\
\hline \multirow{3}{*}{$\begin{array}{l}\text { Seção } 6 \\
\text { Mitigação e Monitoramento }\end{array}$} & Descrição das medidas de mitigação. \\
\hline & Compromisso com mitigação e monitoramento. \\
\hline & Efeitos ambientais da mitigação. \\
\hline $\begin{array}{l}\text { Seção } 7 \\
\text { Resumo não técnico }\end{array}$ & Rima. \\
\hline \multirow[t]{4}{*}{$\begin{array}{l}\text { Seção } 8 \\
\text { Organização e apresentação de } \\
\text { informações }\end{array}$} & $\begin{array}{l}\text { Apresentação de informação (introdução, } \\
\text { apresentação do projeto, objetivos do projeto, } \\
\text { qualidade das informações, definição de termos } \\
\text { técnicos, qualidade dos dados e mapas, qualidade } \\
\text { da linguagem, imparcialidade etc.) }\end{array}$ \\
\hline & $\begin{array}{l}\text { Dificuldades na compilação das informações } \\
\text { (lacunas quanto às informações, dados etc.). }\end{array}$ \\
\hline & Escala das Notas \\
\hline & $\begin{array}{lllll}C & D & E & F & N A\end{array}$ \\
\hline
\end{tabular}

Fonte - Elaborado pelos autores (2020). 
Quadro 4 - Notas atribuídas ao estudo analisado com os critérios de Sánchez (2013).

\begin{tabular}{|c|c|}
\hline $\begin{array}{l}\text { Seção } 1 \\
\text { Sumário }\end{array}$ & $\begin{array}{l}\text { Critério referente à clareza e eficiência do } \\
\text { documento. }\end{array}$ \\
\hline $\begin{array}{l}\text { Seção } 2 \\
\text { Introdução }\end{array}$ & Critério de contextualização do projeto. \\
\hline $\begin{array}{l}\text { Seção } 3 \\
\text { Informações gerais }\end{array}$ & $\begin{array}{l}\text { Critério de qualidade das informações básicas do } \\
\text { empreendimento, legislações, consultas públicas etc. }\end{array}$ \\
\hline $\begin{array}{l}\text { Seção } 4 \\
\text { Caracterização do } \\
\text { empreendimento }\end{array}$ & $\begin{array}{l}\text { Projeto funcional, alternativas locacionais e } \\
\text { tecnológicas etc. }\end{array}$ \\
\hline $\begin{array}{l}\text { Seção } 5 \\
\text { Diagnóstico ambiental }\end{array}$ & $\begin{array}{l}\text { Delimitação da área de estudo, caracterização dos } \\
\text { meios físico, biológico e antrópico, qualidade } \\
\text { ambiental prévia etc. }\end{array}$ \\
\hline $\begin{array}{l}\text { Seção } 6 \\
\text { Análise de impactos }\end{array}$ & $\begin{array}{l}\text { Identificação, previsão e avaliação dos impactos } \\
\text { ambientais, análise dos riscos, prognóstico da } \\
\text { qualidade ambiental futura. }\end{array}$ \\
\hline $\begin{array}{l}\text { Seção } 7 \\
\text { Programas de gestão ambiental }\end{array}$ & $\begin{array}{l}\text { Programas de gestão e plano de monitoramento } \\
\text { ambiental, sistema de gerenciamento ambiental etc. }\end{array}$ \\
\hline $\begin{array}{l}\text { Seção } 8 \\
\text { Síntese e Conclusões }\end{array}$ & Conclusões do EIA, viabilidade ambiental etc. \\
\hline \multirow[t]{3}{*}{$\begin{array}{l}\text { Seção } 9 \\
\text { Rima }\end{array}$} & $\begin{array}{l}\text { Linguagem empregada, concisão, clareza e } \\
\text { relevância das informações apresentadas. }\end{array}$ \\
\hline & Escala das Notas \\
\hline & Completo Aceitável Inadequado \\
\hline
\end{tabular}

Fonte - Elaborado pelos autores (2020).

O Quadro 4 apresenta a análise em relação aos resultados por categoria individual segundo os critérios de Sánchez (2013).

Em relação à avaliação, $67 \%$ dos critérios foram considerados insatisfatórios e $33 \%$ satisfatórios. Somente as seções 1 (sumário) e 2 (introdução) foram atributos completos.

O critério 9 (Rima) foi considerado aceitável, caracterizando incompletude da informação apresentada. Entretanto, todas as outras seções do método foram consideradas inadequadas e apresentando omissões significativas, principalmente as seções referentes à análise dos impactos ambientais.

O Quadro 5 mostra a análise em relação aos resultados por categoria individual segundo os critérios de Pinho, Maia e Monterroso (2005). A avaliação determinou que $25 \%$ dos critérios foram considerados satisfatórios e $75 \%$ insatisfatórios.

Observa-se que critérios importantes, como a caracterização do projeto, a inclusão de alternativas, diagnóstico ambiental, a identificação de impactos, a descrição dos impactos previstos, as medidas mitigadoras, o monitoramento e a participação social foram considerados insatisfatórios, resultado que fragiliza em muito a qualidade do EIA analisado.

$\begin{array}{llllll}\text { Caminhos de Geografia } & \text { Uberlândia-MG } & \text { v. 21, n. 76 } & \text { Ago/2020 } & \text { p. 147-160 Página } 156\end{array}$


Quadro 5 - Notas atribuídas ao estudo analisado com os critérios de Pinho, Maia e Monterroso (2007).

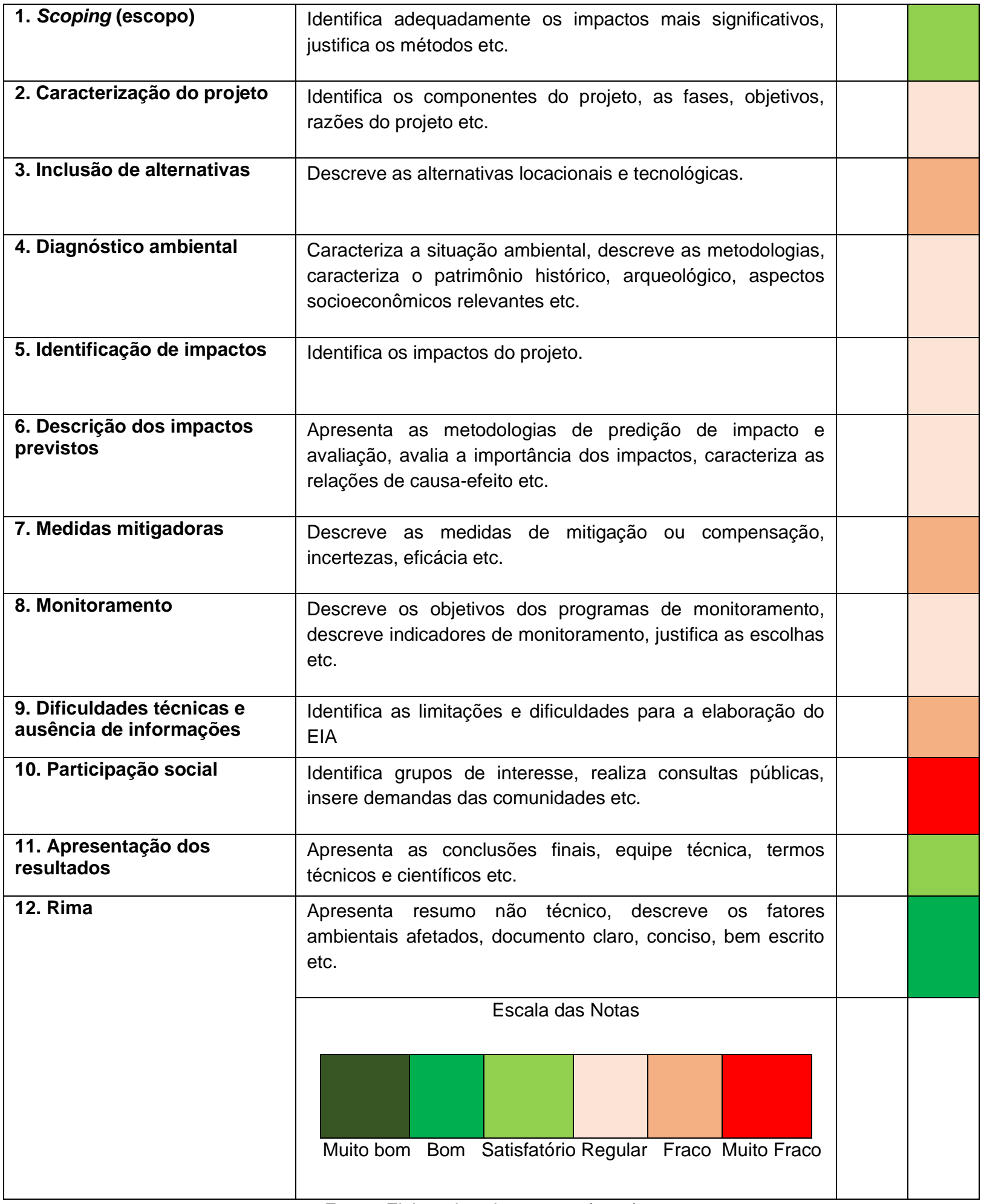

Fonte - Elaborado pelos autores (2020).

A Tabela 1 apresenta uma síntese geral dos resultados obtidos da avaliação do EIA. As quatro ferramentas aplicadas na análise da qualidade do EIA, embora originalmente apresentem diferenças em suas concepções em termos de estrutura geral e de critérios adotados para análise, convergem claramente quanto ao entendimento dos resultados obtidos ao apontarem, sem exceção de nenhum método, para uma faixa que compreende de $61 \%$ a $75 \%$ dos critérios como insatisfatórios, assim mostrando que o EIA é finalmente inadequado (Tabela 1). Tarefas importantes foram omitidas, pontos significativos negligenciados

\begin{tabular}{llllll}
\hline Caminhos de Geografia & Uberlândia-MG & v. 21, n. 76 & Ago/2020 & p. 147-160 & Página 157
\end{tabular}


e questões importantes deixadas de lado, prejudicando em muito a qualidade do estudo e, sobretudo sua análise e consequentemente a tomada de decisão.

Tabela 1 - Síntese da qualidade do EIA pelos métodos empregados no presente trabalho.

\begin{tabular}{ccc}
\hline & \multicolumn{2}{c}{ Resultados dos critérios } \\
& & \\
\hline Métodos & Satisfatórios & Insatisfatórios \\
\hline Lee e Colley (1992) & 35 & 65 \\
Glasson, Therivel e Chadwick & 39 & 61 \\
$(2005)$ & 33 & 67 \\
Sánchez (2013) & 25 & 75 \\
Pinho, Maia e Monterroso & & \\
$(2007)$ & Fonte - Elaborado pelos autores (2020).
\end{tabular}

Com relação às quatro ferramentas usadas neste estudo, as principais diferenças dizem a respeito à estrutura geral e como os critérios são distribuídos ao longo das seções, áreas e categorias de análise.

No geral, a maioria dos critérios adotados de análise são similares. As ferramentas de Lee e Colley (1992), Glasson, Therivel e Chadwick (2005) e Pinho, Maia e Monterroso (2007) são metodologias internacionais, enquanto o método de Sánchez (2013) é uma proposta brasileira adaptada para análise técnica de estudos de impacto ambiental.

Diferenças entre as ferramentas são observadas na definição das escalas de atributos para a avaliação dos critérios.

São comuns às ferramentas os seguintes critérios-chave: diagnóstico ambiental, identificação e análise dos impactos ambientais, medidas mitigadoras, monitoramento ambiental e Rima.

Outros critérios, como por exemplo: descrição do projeto/empreendimento, alternativas locacionais e tecnológicas, síntese e conclusões e informações gerais do projeto/empreendimento, às vezes não estão discriminados na categoria de análise, encontrando-se "diluídos" nas subcategorias.

Observa-se que a maioria dos critérios principais, considerados como elementos fundamentais na análise da qualidade de um EIA, encontram-se presentes nas ferramentas. No entanto, não é possível dizer que existe uma padronização nestes critérios, nem mesmo nas escalas dos atributos para a avaliação destes critérios.

Detectou-se a ocorrência de pequenas discrepâncias na análise de alguns poucos critérios entre os resultados obtidos com as quatro ferramentas, sendo possível citar a descrição, a caracterização do projeto e as condições de linha de base.

Como aspecto positivo, todas as ferramentas consideraram que o Rima foi elaborado de maneira satisfatória.

Ressaltamos que a análise do conteúdo do EIA deve se estabelecer por meio de um processo analítico e sistêmico, em que a fragilidade de um tópico compromete o seguinte e assim por diante, ou seja, um conceito inadequado ou inaceitável a um determinado item ou tópico compromete a qualidade do estudo.

\section{CONSIDERAÇÕES FINAIS}

De um modo geral, apesar de suas diferenças, todas as ferramentas proporcionaram a obtenção de resultados similares, apontando para a baixa qualidade do EIA avaliado. Em uma avaliação geral, o ponto mais fraco identificado no EIA analisado é relacionado ao critério referente à análise dos impactos ambientais.

A avaliação por categoria apontou muitas inadequações no conjunto do estudo avaliado. Os resultados obtidos evidenciam que é necessário melhorar a qualidade dos estudos ambientais e consequentemente do processo de AIA e de licenciamento ambiental.

$\begin{array}{llllll}\text { Caminhos de Geografia } & \text { Uberlândia-MG } & \text { v. 21, n. 76 } & \text { Ago/2020 } & \text { p. 147-160 } & \text { Página } 158\end{array}$


As ferramentas estudadas são úteis para mensurar adequadamente a análise da qualidade de um estudo de impacto ambiental e poderiam ser incorporadas pelos órgãos ambientais licenciadores, como medida de boas práticas e aperfeiçoamento do licenciamento ambiental, auxiliando os técnicos responsáveis pela análise dos estudos ambientais.

Sugere-se que, objetivando a melhoria da qualidade dos EIAs, seria importante estabelecer uma padronização em relação à definição dos critérios de análise e das escalas de atributos para a avaliação destes critérios, bem como exigir, por parte dos órgãos ambientais, maior rigor em relação à qualidade dos estudos de impacto ambiental, rejeitando aqueles que não resultem em uma análise adequada.

\section{REFERÊNCIAS}

AGRA FILHO, S. S. Planejamento e gestão ambiental no Brasil: os instrumentos da política nacional de meio ambiente. 1 ed. Rio de Janeiro: Elsevier, 2014.

ALMEIDA, A. N.; RODRIGUES, N. G.; VIEIRA, L. C. G.; COUTO JUNIOR, A. F. Problemas nos estudos de impacto ambiental de rodovias e ferrovias. Revista Brasileira de Gestão Ambiental e Sustentabilidade, v. 6, n. 12, p. 129-136, 2019. DOI: 10.21438/rbgas.061210

ALMEIDA, M. R. R.; MALFARÁ, D. T.; MENDES, N. C.; MORAES, M. C. P.; SOUZA, M. P. Aplicação de métodos para revisão da qualidade de estudos de impacto ambiental. Revista de Gestão Ambiental e Sustentabilidade, v. 1, n. 2, p. 1-28, 2012. DOI: 10.5585/geas.v1i2.20

ALMEIDA, M. R. R.; MONTAÑO, M. A efetividade dos sistemas de avaliação de impacto ambiental nos estados de São Paulo e Minas Gerais. Ambiente \& Sociedade, v. 20, n. 2, p. 79-106, 2017. https://doi.org/10.1590/1809-4422asoc235r2v2022017

BERTUOLA, H. T.; CANDIANI, G. Métodos para avaliação retrospectiva da qualidade de um estudo de impacto ambiental. Revista Ibero-Americana de Ciências Ambientais, v. 9, n. 8, p. 282-298, 2018. DOI: https://doi.org/10.6008/CBPC2179-6858.2018.008.0025

BONDE, J.; CHERP, A. Quality review package for strategic environmental assessments of land-use plans. Impact Assessment and Project Appraisal, v. 18, n. 2, p. 99-110, 2000. DOI: 10.3152/147154600781767529

CONAMA - Conselho Nacional de Meio Ambiente. Resolução CONAMA no 001, de 23 de janeiro de 1986. Disponível em: http://www2.mma.gov.br/port/conama/legiabre.cfm?codlegi=23 Acesso em: 18 de junho de 2020.

CPA - SP - Coordenação de Planejamento Ambiental - SP. APRM - Billings, Secretaria de Infraestrutura e Meio Ambiente - Governo do Estado de São Paulo. Disponível em: https://www.infraestruturameioambiente.sp.gov.br/cpla/2013/03/aprm-billings/ Acesso em: 04 de junho de 2020.

CPEA - Consultoria, Planejamento e Estudos Ambientais. Estudo de Impacto Ambiental (EIA) do Centro Logístico Campo Grande (CLCG). Paranapiacaba - Santo André, SP, 2017.

GLASSON, J.; THERIVEL, R.; CHADWICK, A. Introduction to Environmental Impact Assessment. 3 ed. London: UCL Press, 2005.

HICKIE, D.; WADE, M. Development of guidelines for improving the effectiveness of environmental assessment. Environmental Impact Assessment Review, v. 18, p. 267-287, 1998. DOI: 10.1016/S01959255(98)00005-5

LEE, N.; COLLEY, R. Reviewing the quality of environmental statements: review methods and findings. Town Plan Rev, v. 62, n. 2, p. 239-248, 1992.

MAZZEI, C. A.; MARANGONI, T. T.; OLIVEIRA, J. N. Análise quantitativa dos estudos de impactos ambientais de hidroelétricas existentes no banco de dados do IBAMA e avaliação dos parâmetros hidrológicos utilizados. Engenharia Sanitária Ambiental, v, 23, n. 3, p. 425-429, 2018. DOI: 10.1590/S1413-41522018169678

MPF - Ministério Público Federal. Deficiências em Estudos de Impacto Ambiental: síntese de uma experiência. Brasília: 4ª Câmara de Coordenação e Revisão, Escola Superior do Ministério Público da União, 2004. 
MONTAÑO, M.; CARVALHO, A. F.; GOMES, C. S.; POLAZ, C. N. M.; JORDÃO, C. O.; SOUZA, M. P. Revisão da qualidade de estudos de impacto ambiental de pequenas centrais hidrelétricas. Holos Environment, v. 14, n. 1, p. 1-14, 2014. DOI: http://dx.doi.org/10.14295/holos.v14i1.6787

MOUNIR, Z. M. Evaluation of the Quality of Environmental Impact Assessment Reports Using Lee and Colley Package in Niger Republic. Modern Applied Science, v. 9, n. 1, p. 89-95, 2015. DOI:10.5539/mas.v9n1p89

PEREIRA, J. A. A.; BORGES, L. A. C.; BARBOSA, A. C. M. C.; BOREM, R. A. T. Fundamentos da Avaliação de Impactos Ambientais com Estudo de Caso. Lavras: UFLA, 2014.

PINHO, P.; MAIA, R.; MONTERROSO, A. The quality of Portuguese Environmental Impact Studies: the case of small hydropower projects. Environmental Impact Assessment Review, v. 27, p. 189-205, 2007. DOI: 10.1016/j.eiar.2006.10.005

PÕDER, T.; LUKKI, T. A critical review of checklist-based evaluation of environmental impact Statements. Impact Assessment and Project Appraisal, v. 29, n. 1, p. 27-36, 2011. https://doi.org/10.3152/146155111X12913679730511

RICCOMINI, B. R. Conflitos e Desafios na Gestão Socioambiental da Região de Paranapiacaba, Santo André-SP. Dissertação (Mestrado em Análise Ambiental Integrada) - Diadema: UNIFESP. 2018.

SÁNCHEZ, L. E. Avaliação de impacto ambiental: conceitos e métodos. 2 ed. São Paulo: Oficina de Textos, 2013.

SCHOEN, C.; SCHULTZ, J.; HEINZ, K.; GROTT, S. C.; PINHEIRO, A. Estudos de Impacto Ambiental: potencialidades, deficiências e perspectivas de elaboradores e avaliadores. Sustentabilidade Em Debate, v. 7, n. 2, p. 257-270, 2016. https://doi.org/10.18472/SustDeb.v7n2.2016.17644

SILVA JUNIOR, L.; ALVARENGA, M. I. N.; GARCIA, S. R. Avaliação da qualidade dos processos de licenciamento ambiental de empreendimentos de mineração em Minas Gerais. Ambiente \& Sociedade, v. 21, p. 1-22, 2018. https://doi.org/10.1590/1809-4422asoc170110r2vu18l1ao

TORRES, P. H. C.; RAMOS, R. F.; GONCALVES, L. R. Conflitos Ambientais na Macrometrópole Paulista: Paranapiacaba e São Sebastião. Ambiente \& Sociedade, v. 22, p.1-18, 2019. https://doi.org/10.1590/18094422asoc20190101vu201912ao

VERONEZ, F.; MONTAÑO, M. Análise da qualidade dos estudos de impacto no estado do Espírito Santo (2007 - 2013). Desenvolvimento e Meio Ambiente, v. 43, p. 6-21, 2017. DOI: 10.5380/dma.v43i0.54180.

Recebido em: 27/12/2019

Aceito para publicação em: 18/06/2020 